\title{
Improving CCT-D and LO of the 6600K ICP-WLEDs by $\mathrm{K}_{2} \mathrm{SiF}_{6}: \mathrm{Mn}^{4+}$ phosphor
}

\author{
Phu Tran Tin', Le Anh Vu², Minh Tran³, Nguyen Huu Khanh Nhan ${ }^{4}$, Tran Thanh Trang ${ }^{5}$ \\ ${ }^{1}$ Faculty of Electronics Technology, Industrial University of Ho Chi Minh City, Vietnam \\ ${ }^{2,3,4}$ Optoelectronics Research Group, Faculty of Electrical and Electronics Engineering, Ton Duc Thang University, Ho \\ Chi Minh City, Vietnam \\ ${ }^{5}$ Faculty of Engineering and Technology, Van Hien University, 665-667-669 Dien Bien Phu, Ho Chi Minh City, \\ Vietnam
}

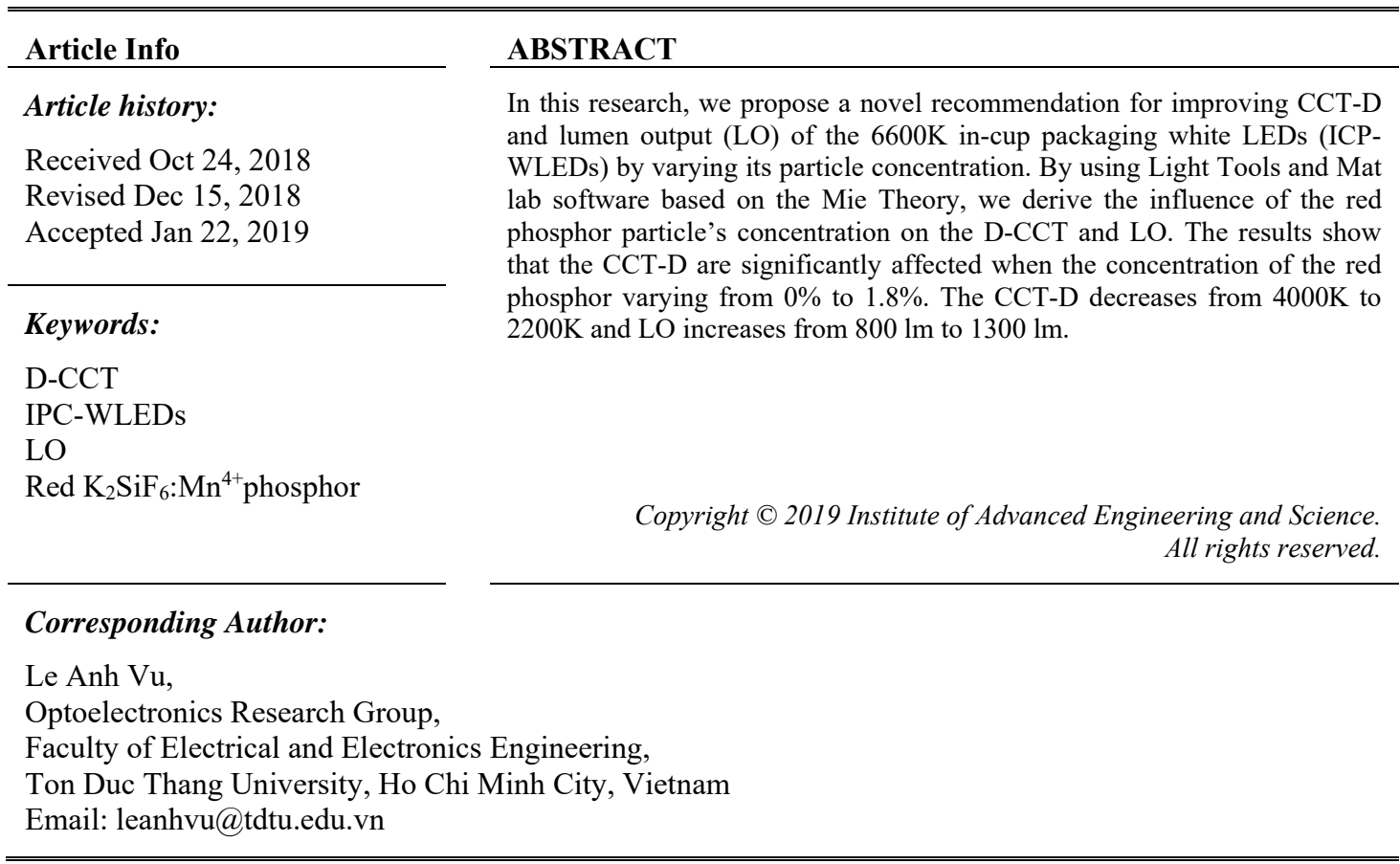

\section{INTRODUCTION}

From the first invented light emitting diodes (LEDs) by H. J. Round in 1907, LEDs is considered as the future lighting generation. After Nick Holonyak invented the LEDs in 1962, LEDs was focused more and more attention by the researchers around the world. LEDs with high brightness and blue light was proposed in 1995, which made it possible to use LEDs for general lighting in industrial and civil purpose. Nowadays, LEDs is considered as the fourth lighting generation technology in competition with the traditional incandescent and (compact) fluorescent lamps and can be replaced with the old ones in our time because they have excellent advantages such as low electric consumption, high brightness, long lifetime, small size, robustness, fast switching, and environment-friendly characters [1-4]. From many types of research in this field, we can see that material and packaging is the leading research direction on improving the optical performance of the LEDs [5-7]. Authors in [8] stated that not only method packaging but also concentration, size, and thickness of the phosphor layer is the main effect factor on the luminous efficacy and color qualities of the white LEDs. Such as, [9] considered the effects of phosphor thickness and concentration on LEDs luminous flux and correlated color temperature by the experimental method. In [10], the effect of phosphor location on the spatial color distribution was demonstrated. In this trend, authors in [11-12] investigated the effect of the red-emitting phosphor on the lighting performance and optical properties of the white LEDs. For extending the research in [13], this paper investigated the influence of the red phosphor concentration on the color correlated temperature deviation (CCT-D) and lumen output (LO) of the white LEDs. 
As rare-earth-free alternatives, the manganese-based phosphors K2SiF6:Mn4+ has d-d transitions, which are partially spin-allowed for Mn4+. Upon incorporation in the host compound, Mn4+ is the preferred valence state for LED applications, due to better excitation properties and narrower emission bands. In K2SiF6:Mn4+, the Intra configurational 3d3 transitions of the $\mathrm{Mn} 4+$ ion are responsible for the luminescence, yielding narrow line emission. Furthermore, K2SiF6:Mn4+ has the relatively high values of the color quality scale (CQS), color purity and absorption strength. The specific red line emission is seen in K2SiF6:Mn4+ around $630 \mathrm{~nm}$ is caused by 3d3-3d3 transitions in Mn4+.The $3 \mathrm{~d} 3$ electron configuration of Mn4+ results in 120 possible distributions of the three electrons over the ten available single-particle states of the $3 \mathrm{~d}$ shell. The electronic Coulomb repulsion gives rise to a splitting of the 120 -fold degenerate configuration in eight LS terms that can be found for the free Mn4+ ion, the magnitude of this splitting is characterized by the Racah parameters B and C [13-14]. This red phosphor can be considered as the prospective candidate for improving color quality of the white LEDs.

In this research, the red-emitting $\mathrm{K} 2 \mathrm{SiF} 6: \mathrm{Mn} 4+$ conversion phosphor is considered as a novel recommendation for increasing the CCT-D and LO of the $6600 \mathrm{~K}$ in-cup packaging white LEDs (ICPWLEDs). The effect of the red-emitting K2SiF6:Mn4+ conversion phosphor is investigated based on Mie Theory with helping of the Mat Lab and Light Tools software. The research results show that the CCT deviation (CCT-D), lumen output (LO) has been remarkably affected while the concentration of the redemitting K2SiF6:Mn4+ conversion phosphor's particle varies. Finally, the red-emitting K2SiF6:Mn4+ conversion phosphor can be considered as a novel recommendation for WLEDs manufacturing. The main contributions of this manuscript can be summarized as the followings:

a. The physical model and mathematical description of the ICP-WLEDs is conducted and formulated by Mat Lab and Light Tools software based on Mie Theory.

b. The scattering process in the phosphor layer of the ICP-WLEDs is investigated.

c. The CCT-D and LO are analyzed and discussed in connection with varying the concentration of the red phosphor particles.

\section{RESEARCH METHOD}

In this research, we use the real model of WLEDs as shown in Figure 1(a) for simulating the physical model of ICP-WLEDs as shown in Figure 1(b). In this simulation model, we set the main parameters of the $6600 \mathrm{~K}$ CP-WLEDs as;

a. The depth, the inner and outer radius of the reflector to $2.07 \mathrm{~mm}, 8 \mathrm{~mm}$ and $9.85 \mathrm{~mm}$, respectively.

b. LEDs chips are covered with a fixed thickness of $0.08 \mathrm{~mm}$ and $2.07 \mathrm{~mm}$.

c. Each blue chip has a dimension of $1.14 \mathrm{~mm}$ by $0.15 \mathrm{~mm}$, the radiant flux of $1.16 \mathrm{~W}$, and the peak wavelength of $453 \mathrm{~nm}$ as shown in Figure 1(b) [11-13].

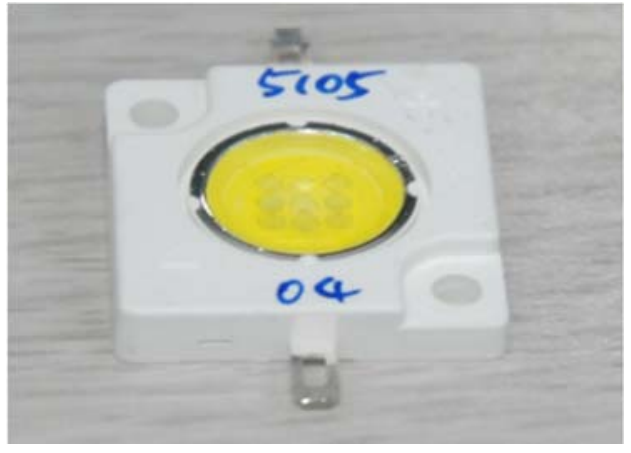

(a)

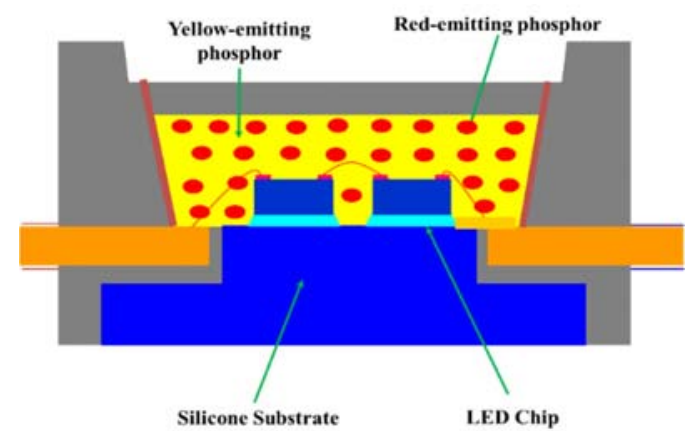

(b)

Figure 1. (a) The WLEDs product of the Siliconware Precision Industries Co., Ltd.,Taiwan, (b) ICP-WLEDs

For demonstrating the influence of the red phosphor's concentration on the CCT-D and LO, we can simulate the scattering and reduced scattering processes inside the phosphor layer of the 6600K ICP-WLEDs based on the Mie-scattering [15-20]. The below expressions can compute the scattering coefficient $\mu \mathrm{sca}(\lambda)(\mathrm{mm}-1)$, and reduced scattering coefficient $\delta \operatorname{sca}(\lambda)(\mathrm{mm}-1)$. 


$$
\begin{aligned}
& \mu_{s c a}(\lambda)=\int N(r) C_{s c a}(\lambda, r) d r \\
& \delta_{s c a}=\mu_{s c a}(1-g)
\end{aligned}
$$

In these equations, $N(r)$ indicates the distribution density of diffusional particles $\left(\mathrm{mm}^{3}\right) . C_{s c a}$ is the scattering cross sections $\left(\mathrm{mm}^{2}\right), \lambda$ is the light wavelength $(\mathrm{nm}), r$ is the radius of diffusional particles $(\mu \mathrm{m})$, Moreover, $N(r)$ can be calculated by:

$$
N(r)=N_{d i f}(r)+N_{p h o s}(r)=K_{N} \cdot\left[f_{d i f}(r)+f_{p h o s}(r)\right]
$$

$N(r)$ is composed of the diffusive particle number density $N_{d i f}(r)$ and the phosphor particle number density $N_{\text {phos }}(r)$. In these equations, $f_{\text {dif }}(r)$ and $f_{\text {phos }}(r)$ are the size distribution function data of the diffusor and phosphor particle. Here $K_{N}$ is the number of the unit diffusor for one diffuser concentration and can be calculated by the following equation:

$$
c=K_{N} \int M(r) d r
$$

Where $M(r)$ is the mass distribution of the unit diffuser and can be proposed by the below equation:

$$
M(r)=\frac{4}{3} \pi r^{3}\left[\rho_{\text {dif }} f_{d i f}(r)+\rho_{\text {phos }} f_{\text {phos }}(r)\right]
$$

Here $\rho_{\text {diff }}(r)$ and $\rho_{\text {phos }}(r)$ are the density of diffuser and phosphor crystal.

\section{RESULTS AND DISCUSSION}

In this section, the influence of the red phosphor particles' concentration on the scattering process inner the phosphor layer of the 6600K ICP-WLEDs is investigated using Mat Lab software in the first stage. After that, the CCT-D and LO of the 6600K ICP-WLEDs are analyzed and discussed in connection with varying the concentration of the red phosphor from $0 \%$ to $1.8 \%$. The scattering and reduced scattering processes inner phosphor layer are drawn in Figure 2 and 3. Figure 2 indicates that the scattering coefficient of $453 \mathrm{~nm}, 55 \mathrm{~nm}$, and $680 \mathrm{~nm}$ significantly increase while the concentration of the red phosphor varying from $1 \%$ to $5 \%$. In addition, the coefficient of $555 \mathrm{~nm}$ has the highest increase in comparison with others. On the same way, the reduced scattering coefficients of $453 \mathrm{~nm}, 55 \mathrm{~nm}$, and $680 \mathrm{~nm}$ have a considerable increase while the concentration of the red phosphor varying from $1 \%$ to $5 \%$. All $453 \mathrm{~nm}, 55 \mathrm{~nm}$, and 680 $\mathrm{nm}$ reduced scattering coefficients increase in the same direction with each other as shown in Figure 3 . From that point of view, it is observed that the scattering process in the phosphor layer of the 6600K ICP-WLEDs can be controlled by varying the concentration of the red phosphor.

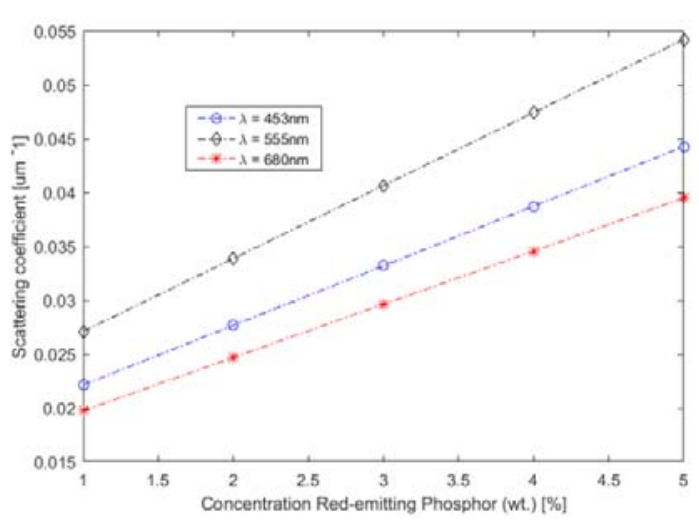

Figure 2. Scattering coefficients

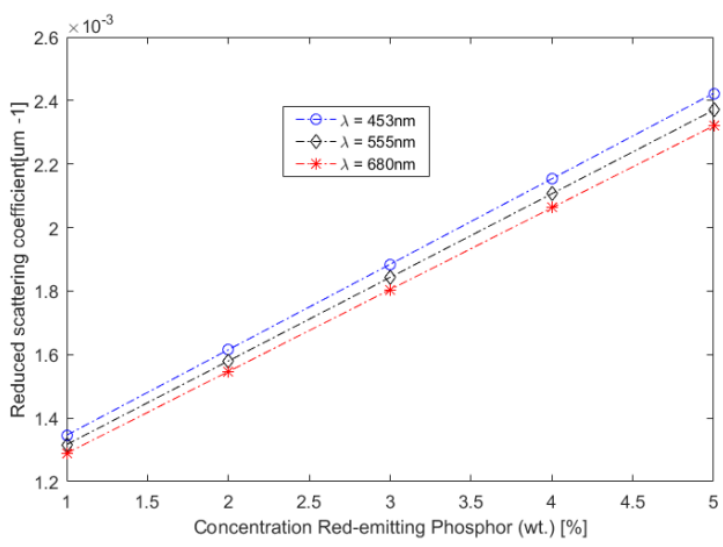

Figure 3 Reduced scattering coefficient 
Moreover, the CCT-D and LO versus the red phosphor particles' concentration are illustrated in Figure 4 and 5 . In this analysis, the concentration of the red phosphor is controlled from $0 \%$ to $1.8 \%$. From the research results, we can see that the CCT-D has a huge decrease and the LO has a massive increase with increasing the concentration of the red phosphor. When the concentration of the red phosphor from $0 \%$ to $1.8 \%$, CCT-D decreases from $4000 \mathrm{~K}$ to $2200 \mathrm{~K}$ and LO increase from $8001 \mathrm{~m}$ to $1300 \mathrm{~lm}$. It can be observed that we can improve the CCT-D and LO of the 6600K ICP-WLEDs by controlling the concentration of the red phosphor particles.

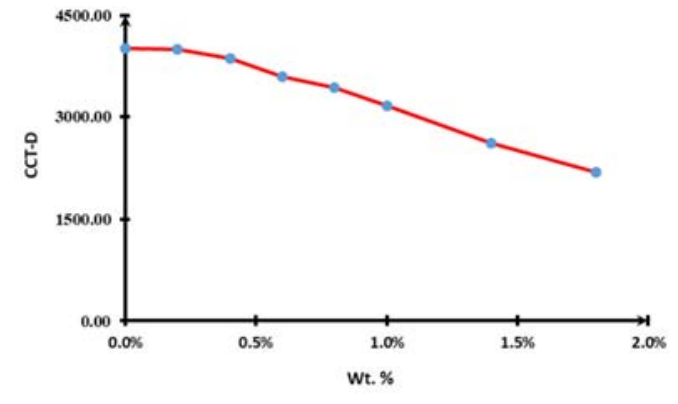

Figure 4. CCT-D versus Wt.\%

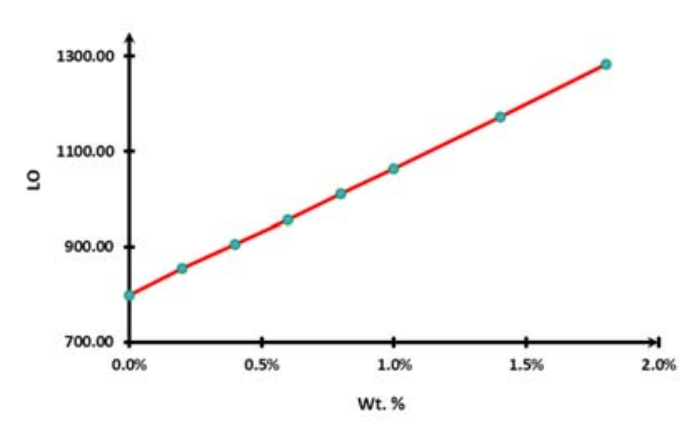

Figure 5. LO versus Wt. \%

\section{CONCLUSION}

In this research, a novel recommendation for improving CCT-D and LO of the 6600K ICP-WLEDs by varying its particle concentration is considered. By using Light Tools and Mat lab software based on the Mie Theory, we derive the influence of the red phosphor particle's concentration on the D-CCT and LO. The results show that the CCT-D are significantly affected when the concentration of the red phosphor varying from $0 \%$ to $1.8 \%$. The CCT-D decreases from $4000 \mathrm{~K}$ to $2200 \mathrm{~K}$ and LO increases from $800 \mathrm{~lm}$ to $1300 \mathrm{~lm}$.

\section{REFERENCES}

[1] Sheng Liu Xiaobing Luo. "LED Packaging for Lighting Applications", Design of LED Packaging Applications, 215-315. 20 July 2011 doi:10.1002/9780470827857.ch6.

[2] Gibney, Elizabeth. "Nobel for Blue LED That Revolutionized Lighting." Nature. Vol. 514 No. 7521, pp. 152-153. 2014. doi:10.1038/514152a.

[3] Luo, Xiaobing, Run Hu, Sheng Liu, and Kai Wang. "Heat and Fluid Flow in High-Power LED Packaging and Applications." Progress in Energy and Combustion Science, Vol 56, pp. 1-32. 2016. doi:10.1016/j.pecs.2016.05.003.

[4] Winkler, Holger, Quang Trinh, Peter Bodrogi, and Tran Quoc Khanh. LED Lighting:Technology and Perception. Weinheim: Wiley-VCH, 2015.

[5] Smet, P. F., Parmentier, A. B., \& Poelman, D. "Selecting Conversion Phosphors for White Light-Emitting Diodes". Journal of the Electrochemical Society, vol 158, no 6, 2011. doi:10.1149/1.3568524

[6] Y. Shuai, Y. He, N. T. Tran and F. G. Shi, "Angular CCT Uniformity of Phosphor Converted White LEDs: Effects of Phosphor Materials and Packaging Structures," in IEEE Photonics Technology Letters, vol. 23, no. 3, pp. 137139, Feb.1, 2011.

[7] Z. Liu, S. Liu, K. Wang and X. Luo, "Optical Analysis of Color Distribution in White LEDs With Various Packaging Methods," in IEEE Photonics Technology Letters, vol. 20, no. 24, pp. 2027-2029, Dec.15, 2008.

[8] Liu, Zongyuan, Sheng Liu, Kai Wang, and Xiaobing Luo. "Effects of Phosphor's Location on LED Packaging Performance." 2008 International Conference on Electronic Packaging Technology \&amp; High Density Packaging, 2008. doi:10.1109/icept.2008.4606982.

[9] Tran, Nguyen T., and Frank G. Shi. "Studies of Phosphor Concentration and Thickness for Phosphor-Based White Light-Emitting-Diodes." Journal of Lightwave Technology, Vol 26, no. 21, pp. 3556-3559, 2008. doi:10.1109/jlt.2008.917087.

[10] Liu, Zongyuan, Sheng Liu, Kai Wang, and Xiaobing Luo. "Effects of Phosphor's Location on LED Packaging Performance." 2008 International Conference on Electronic Packaging Technology \&amp; High Density Packaging. 2008. doi:10.1109/icept.2008.4606982.

[11] Minh, Tran Hoang Quang, Nguyen Huu Khanh Nhan, Nguyen Doan Quoc Anh, and Hsiao-Yi Lee. "Red-Emitting $\alpha$-SrO-3B2O3:Sm2 Phosphor: an Innovative Application for Increasing Color Quality and Luminous Flux of Remote Phosphor White LEDs.” Journal of the Chinese Institute of Engineers, Vol 40, no. 4, pp. 313-317, 2017. doi:10.1080/02533839.2017.1318720.

Int J Pow Elec \& Dri Syst, Vol. 10, No. 2, June 2019 : 1059 - 1063 
[12] Anh, Nguyen Doan Quoc, Hsiao-Yi Lee, Tran Thanh Phuong, Nguyen Huu Khanh Nhan, Tran Hoang Quang Minh, and Truong Huu Ly. "Y2O3:Eu3 Phosphor: a Novel Solution for an Increase in Color Rendering Index of Multi-Chip White LED Packages.” Journal of the Chinese Institute of Engineers, vol. 40, no. 3, pp. 228-234, 2017. doi:10.1080/02533839.2017.1299592.

[13] Tin, Phu Tran, Nguyen Huu Khanh Nhan, and Tran Hoang Quang Minh. "Increasing the Color Quality of the 7000K Conformal Packaging MCW-LEDs by Varied Red-emitting K2SiF6:Mn4 Conversion Phosphor's Size." Cogent Engineering, vol. 4, no. 1, 2017. doi:10.1080/23311916.2017.1404718.

[14] Sijbom, H. F., Verstraete, R., Joos, J. J., Poelman, D., \& Smet, P. F. "K_2SiF_6:Mn^4 as a red phosphor for displays and warm-white LEDs: A review of properties and perspectives". Optical Materials Express, vol 7, no. 9, pp.3332, 2017. doi:10.1364/ome.7.003332

[15] Michael Quinten Optical Properties of Nanoparticle Systems. "Beyond Mie's Theory II - The Generalized Mie Theory," 317-339, 2011. doi:10.1002/9783527633135.ch10.

[16] Frisvad, Jeppe Revall, Niels Jørgen Christensen, and Henrik Wann Jensen. "Predicting the Appearance of Materials Using Lorenz-Mie Theory." The Mie Theory Springer Series in Optical Sciences, pp. 101-133, 2012. doi:10.1007/978-3-642-28738-1_4.

[17] Mackowski, Daniel. "The Extension of Mie Theory to Multiple Spheres." The Mie Theory Springer Series in Optical Sciences, pp. 223-256, 2012. doi:10.1007/978-3-642-28738-1_8.

[18] Wriedt, Thomas. 2012. "Mie Theory: A Review." The Mie Theory Springer Series in Optical Sciences, 53-71. doi:10.1007/978-3-642-28738-1_2.

[19] K. Pan, et al., "Study on Reliability and Lifetime Prediction of High Power LEDs," Indonesian Journal of Electrical Engineering and Computer Science (IJEECS), Vol 12, no. 2, pp. 1132-1142, February 2014.

[20] Peng H. Y., et al., "Red (SrCa)AlSiN3: Eu2+ Nitride Phosphor Particle Size of Phosphor Converted Warm White LEDs," Indonesian Journal of Electrical Engineering and Computer Science (IJEECS). vol 12, no 7, pp.52115216, July 2014. 